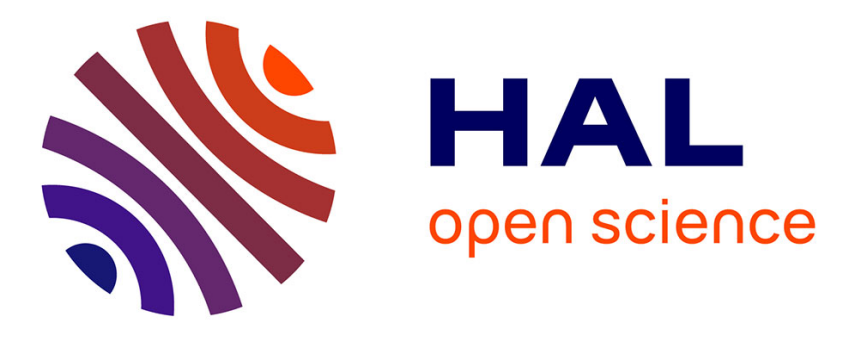

\title{
Identification of Chirps with Continuous Wavelet Transform
}

René Carmona, Wen-Liang Hwang, Bruno Torrésani

\section{To cite this version:}

René Carmona, Wen-Liang Hwang, Bruno Torrésani. Identification of Chirps with Continuous Wavelet Transform. Anestis Antoniadis, Georges Oppenheim. Wavelets and Statistics, 103, Springer, pp.95-108, 1995, Lecture Notes in Statistics, 978-0-387-94564-4. 10.1007/978-1-4612-2544-7_7 . hal01304136

\section{HAL Id: hal-01304136 https://hal.science/hal-01304136}

Submitted on 19 Apr 2016

HAL is a multi-disciplinary open access archive for the deposit and dissemination of scientific research documents, whether they are published or not. The documents may come from teaching and research institutions in France or abroad, or from public or private research centers.
L'archive ouverte pluridisciplinaire HAL, est destinée au dépôt et à la diffusion de documents scientifiques de niveau recherche, publiés ou non, émanant des établissements d'enseignement et de recherche français ou étrangers, des laboratoires publics ou privés. 


\title{
Identification of Chirps with Continuous Wavelet Transform
}

\author{
René Carmona ${ }^{1}$, Wen Liang Hwang ${ }^{1}$ and Bruno Torrésani ${ }^{2}$ \\ 1 Department of Mathematics, University of California at Irvine, Irvine, CA 92717, USA \\ 2 CPT, CNRS-Luminy, Case 907, 13288 Marseille Cedex 09,FRANCE
}

\begin{abstract}
Chirps are signals (or sums of signals) that may be characterized by a local (i.e. time-dependent) amplitude and a local frequency. Time-frequency representations such as wavelet representations are well adapted to the characterization problem of such chirps. Ridges in the modulus of the transform determine regions in the transform domain with a high concentration of energy, and are regarded as natural candidates for the characterization and the reconstruction of the original signal. A couple of algorithmic procedures for the estimation of ridges from the modulus of the (continuous) wavelet transform of one-dimensional signals are described, together with a new reconstruction procedure, using only information of the restriction of the wavelet transform to a sample of points from the ridge. This provides with a very efficient way to code the information contained in the signal.
\end{abstract}

\section{Generalities}

There exists a large class of signals that may be modeled as sums of amplitude and frequency modulated components, i.e. in the form

$$
f(x)=\sum_{k} A_{k}(x) \cos \phi_{k}(x)
$$

where the relative variations of the amplitudes are assumed to be small compared with the oscillations, and the local frequencies

$$
\nu_{k}(x)=\frac{1}{2 \pi} \phi_{k}^{\prime}(x)
$$

are assumed to be slowly varying.

The characterization of such signals and the separation of their components (in the presence of noise) is a classical problem of signal analysis and signal processing, that goes back to pioneering work of J. Ville [16]. Applications can be found in many situations, such as for instance radar/sonar detection and speech processing [13]. Clearly, time-frequency methods (linear methods such as wavelet or short time Fourier transforms, or bilinear methods such as Wigner distributions) can provide satisfactory answers, at least in some situations and for large values of the signal to noise ratio. 
The main point of the analysis is the following heuristics: Let $f(x)=A(x) \cos \phi(x)$ be a real-valued function. Time-frequency representations of $f(x)$ happen to "concentrate" in regions of the time-frequency plane determined by the instantaneous frequency $\phi^{\prime}(x)$. One may then use the time-frequency localization of these representations to obtain a characterization of this class of signals (see Flandrin's book [9] for a review).

A family of algorithms was proposed in 1990 by the group of Marseille (see [8] and [15] for a survey). These algorithms may be called "differential methods", in the sense that they are based on a local study of the variations of the modulus and the phase of the continuous wavelet (or Gabor) transform. In a noisy situation, it is however necessary to use additional informations to stabilize the estimation. We shall describe here two methods in which the a-priori information (namely the smoothness of the ridge, consequence of the slowly varying character of the frequencies, and the slow variations of the amplitudes) is taken into account. Our approach (developed in [5]) combines the wavelet transform with a simulated annealing algorithm [10], and is an "integral-type method". We also propose a stable method (also based on an optimization procedure) for signal reconstruction from the numerically computed ridges.

For the sake of simplicity, our discussion will be restricted to the case of the wavelet transform. But it is important to notice that since our algorithms deals only with postprocessing of time-frequency transforms, they can be extended to any time-frequency energetic representations (see for example [6] where another stochastic search algorithm based on Gabor transform is introduced).

Our notations and conventions are as follows. We shall work with the $L^{2}(\mathbb{R})$ setting, and we shall concentrate on the case of the continuous (complex-valued) wavelet transform. If $\psi \in L^{1}(\mathbb{R})$ is such that:

$$
0<c_{\psi}=\int_{0}^{\infty}|\hat{\psi}(\xi)|^{2} \frac{d \xi}{\xi}<\infty
$$

(where the convention for the Fourier transform is $\hat{f}(\xi)=\int_{-\infty}^{\infty} f(x) e^{-i \xi x} d x$ ), i.e. fulfills the wavelet admissibility condition, then the wavelet transform of a signal $f(x)$ with respect to $\psi$ is given by:

$$
T_{f}(b, a)=\frac{1}{a} \int_{-\infty}^{\infty} f(x) \psi\left(\frac{x-b}{a}\right)^{*} d x .
$$

We will focus on the case of complex-valued wavelets (namely we assume that $\psi(x) \in$ $H^{2}(\mathbb{R})=\left\{f \in L^{2}(\mathbb{R}), \hat{f}(\xi)=0 \forall \xi \leq 0\right\}$ and we set:

$$
\psi_{(b, a)}(x)=\frac{1}{a} \psi\left(\frac{x-b}{a}\right) .
$$

The synthesis from wavelet transform is expressed as follows. If $f \in H^{2}(\mathbb{R})$ :

$$
f=\frac{1}{c_{\psi}} \int_{0}^{\infty} \int_{\mathbb{R}} T_{f}(b, a) \psi_{(b, a)} d b \frac{d a}{a} .
$$

Since a real-valued signal is completely characterized by its positive frequencies, the case of real-valued signals is handled similarly. 


\section{Ridge detection from the wavelet transform modulus}

The keystones of our descriptions will be the "wavelet Plancherel formula"

$$
\left\|T_{f}\right\|_{L^{2}\left(R_{+} \times \mathbb{R}\right)}^{2}=c_{\psi}\|f\|^{2}, \quad f \in L^{2}(\mathbb{R}) .
$$

which allows us to interpret the square-modulus of the continuous wavelet transform as a "time-scale" energy density, and the following lemma

Lemma 2.1 Let $f(x)=A(x) \cos \phi(x)$, and let $T_{f}(b, a)$ be its wavelet transform. Then

$$
T_{f}(b, a)=\frac{1}{2} A(b) e^{i \phi(b)} \hat{\psi}\left(a \phi^{\prime}(b)\right)^{*}+O\left(\left|A^{\prime}\right|,\left|\frac{\phi^{\prime \prime} \phi}{\phi^{\prime 2}}\right|\right)
$$

which expresses the fact that as long as the remainder can be neglected, the wavelet transform is essentially concentrated in a neighborhood of a curve of the form

$$
a=\varphi(b)=\frac{\omega_{0}}{\phi^{\prime}(b)}
$$

for some constant $\omega_{0}$ determined by the wavelet $\psi(x)$ (the so-called central frequency of the wavelet). Characterizing the signal's instantaneous frequency by the wavelet transform can then be achieved (within certain accuracy) by extracting numerically the ridge as a set of local extrema of the modulus of the wavelet transform.

\subsection{Ridge estimation}

Let us now turn to the case of noisy signals. Let then

$$
f(x)=A(x) \cos \phi(x)+\text { noise }
$$

be our noisy signal, and let $T_{f}(b, a)$ be its continuous wavelet transform. It is clear that for significantly negative values of the SNR, both the modulus and the phase can be corrupted by the noise. In order to characterize the signal, we introduce more rigidity into the method, by implementing the a-priori information that the ridge has to be a smooth function of the time variable.

Denote by $\Phi$ the space of all the smooth (say twice continuously differentiable functions with square integrable derivatives) functions. We then introduce the following penalty function $F_{f}$ on the set $\Phi$ of ridge candidates $\varphi$ :

$$
F_{f}(\varphi)=-\int\left|T_{f}(b, \varphi(b))\right|^{2} d b+\int\left[\lambda \varphi^{\prime}(b)^{2}+\mu \varphi^{\prime \prime}(b)^{2}\right] d b
$$

Our estimate of the unknown ridge of the wavelet transform of the signal $f$ will be the function $a=\varphi(b)$ that minimizes $F_{f}(\varphi)$. It is not very difficult to obtain the Euler equation associated with this minimization problem (see [5]), and to solve it numerically, say by some finite differences method. However, the presence of noise may result in the existence of many local extrema in the wavelet transform modulus, so that any standard algorithm may be trapped in local extrema of $F_{f}(\varphi)$. At this point an alternative is provided by a simulated annealing algorithm (see e.g. [10] [12]) which can jump over the local extrema to reach the global one(s). 
2.1.1 The algorithm The first step is a discretization of the problem. Assuming that we start from a wavelet transform defined for the discrete grid $[0,1, \ldots, B-1] \times[0,1, \ldots, A-1]$ in the time-scale plane, the ridge will take the form of a finite sequence:

$$
\{\varphi(0), \varphi(1), \ldots \varphi(\ell), \ldots \varphi(n-1)\}
$$

We then need to specify a neighborhood system for our discretized ridges. The neighbors we shall consider are the simplest possible ones. The set $N(\varphi)$ of neighbors of $\varphi$ is the set of finite sequences:

$$
\{\varphi(0), \varphi(1), \ldots \varphi(\ell) \pm 1, \ldots \varphi(n-1)\}
$$

with $\ell=0, \ldots n-1$.

Our algorithm is then given by the following iteration:

- Initialization: Start from a given value $T_{0}=C / \ln (2)$ of the temperature and from an initial guess: $\left\{\varphi_{0}(0), \varphi_{0}(1), \ldots \varphi_{0}(n-1)\right\}$ and compute $F_{f}\left(\varphi_{0}\right)$.

- Step $k$ : If the ridge at step $k-1$ is known, say:

$$
\varphi_{k-1}=\left\{\varphi_{k-1}(0), \varphi_{k-1}(1), \ldots \varphi_{k-1}(n-1)\right\} .
$$

- Update the temperature with a given schedule, for instance (see [12] for a discussion of other possible schedules):

$$
T_{k}=\frac{C}{\ln (1+k)}
$$

- Generate randomly (with uniform probability) an integer $\ell \in[0, n-1]$ and a number $\varepsilon= \pm 1$. The candidate $\varphi_{k}^{c}$ for step $k$ is then

$$
\varphi_{k}^{c}=\left\{\varphi_{k}^{c}(0), \varphi_{k}^{c}(1), \ldots \varphi_{k}^{c}(\ell)+\varepsilon, \ldots \varphi_{k}^{c}(n-1)\right\} \text {. }
$$

- Compute the value of the cost function $F_{f}\left(\varphi_{k}^{c}\right)$ and compare it with $F_{f}\left(\varphi_{k-1}\right)$.

- If $F_{f}\left(\varphi_{k}^{c}\right) \leq F_{f}\left(\varphi_{k-1}\right)$ update the ridge: the new ridge at step $k$ is $: \varphi_{k}=\varphi_{k}^{c}$.

- If $F_{f}\left(\varphi_{k}^{c}\right)>F_{f}\left(\varphi_{k-1}\right)$ pick a random number $\sigma$ uniformly distributed between 0 and 1 .

$*$ If $\sigma \leq \exp \left\{-\left[F_{f}\left(\varphi_{k}\right)-F_{f}\left(\varphi_{k-1}\right)\right] / T_{k}\right\}$ update the ridge: $\varphi_{k}=\varphi_{k}^{c}$.

$*$ otherwise keep the previous ridge: $\varphi_{k}=\varphi_{k-1}$.

- Stopping criterion: When the ridge has not changed for a certain number of steps (fixed in advance), stop the iteration (see [12] for a discussion of some possible stopping criteria).

Remark: In practice it is useful to run this algorithm on a "smoothed+subsampled" version of the wavelet transform $T(b, a)$, in order to reduce the computational cost of the algorithm. 
2.1.2 Pre-denoising In some situations, some "a-priori" knowledge of the noise is available. We can for instance quote the cases where the power spectrum of the noise is known in advance, or the cases where a piece of the signal is known to contain only noise (which gives us the chance to learn about the statistics of this noise). Then one can "correct" the wavelet transform, by substracting what is supposed to be the "average" contribution of the noise. This contribution could be chosen to be the expectation $\mathbb{E}\left[\left|T_{n}(b, a)\right|^{2}\right]$ over all the possible realizations of the additive noise, say $n$. If an a-priori model for the noise is available, such a quantity may be estimated by Monte-Carlo simulations, or sometimes by a direct computation For instance, in the case of a second order stationary noise, with power spectrum of the form $p(\xi)=\sigma^{2} \xi^{\alpha}$, it is easy to derive that $\mathbb{E}\left[\left|T_{n}(b, a)\right|^{2}\right] \sim K_{\alpha} \sigma^{2} a^{-\alpha-1}$; for this the wavelet $\psi(x)$ has to be such that $K_{\alpha}=\int u^{\alpha}|\hat{\psi}(u)|^{2} d u<\infty$.

In the general case, we only have one realization of the noise at hand, and it is impossible to compute directly this expectation .... But a simple ergodic argument justifies the use of the estimate:

$$
V(a)=\frac{1}{B} \int_{0}^{B}\left|T_{n}(b, a)\right|^{2} d b .
$$

Then the cost function given in equation (11) may be replaced by

$$
\tilde{F}_{f}[\varphi]=-\int\left[\left|T_{f}(b, \varphi(b))\right|^{2}-V(\varphi(b))\right] d b+\int\left[\lambda \varphi^{\prime}(b)^{2}+\mu \varphi^{\prime \prime}(b)^{2}\right] d b
$$

Such a modification sometimes avoids "trapping" the ridge in regions dominated by the noise. In the numerical experiments presented in [5], this modified cost function allowed us to handle values of the SNR up to $-6 d B$. Of course there exists other possibilities for a-priori models of the noise, which may be implemented in our scheme in a simple way.

\subsection{Illustration}

Detailed illustration of the algorithms described here may be found in [5], where real signals corrupted by Gaussian noise with various values of the SNR are presented.

We only exhibit here simple examples, based on an academic signal, a simple model for a whistle with time-varying tone. We present in figure 2 the modulus (coded with gray levels) of the wavelet transform of the chirp signal shown in figure 1 and at the top of figure 2 (the instantaneous frequency is a linear function of time, and the amplitude is a Gaussian function), together with the associated ridge (the analyzing wavelet is in this case the Morlet wavelet $\psi(x)=\exp \left\{-x^{2} / 2\right\} \exp \left\{i \omega_{0} x\right\}$ with $\omega_{0}=2 \pi$, which is not strictly speaking an admissible wavelet, but is such that $\hat{\psi}(0)$ is small enough to be neglected in double precision calculations). The ridge is easily seen to localize in the most energetic region of the time-frequency plane. More precisely it reproduces the law given by equation (9), and the results of the algorithm are seen to be very close to those obtained with previously developed methods (see [8]).

A similar analysis was done on a noisy copy of the chirp signal (the noise was an uniform white noise, with signal to noise ratio around $0 d B$; we used the penalty function $F_{f}[\varphi]$ and not $\tilde{F}_{f}[\varphi]$ ). The results are presented in figure 3 (the noisy signal is displayed at the top of the figure). The ridge is detected correctly, although the wavelet transform can be seen to be dramatically perturbed by the noise; this is the effect of the smoothness penalty of the cost function. 


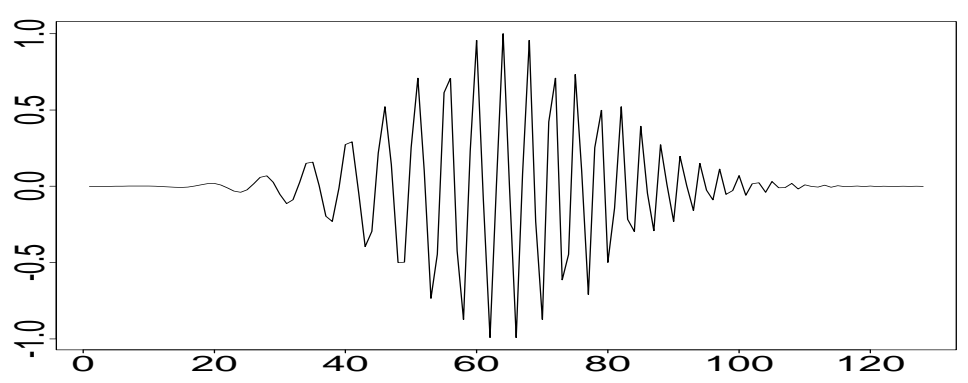

Figure 1: Chirp signal used to illustrate the reconstruction procedure.

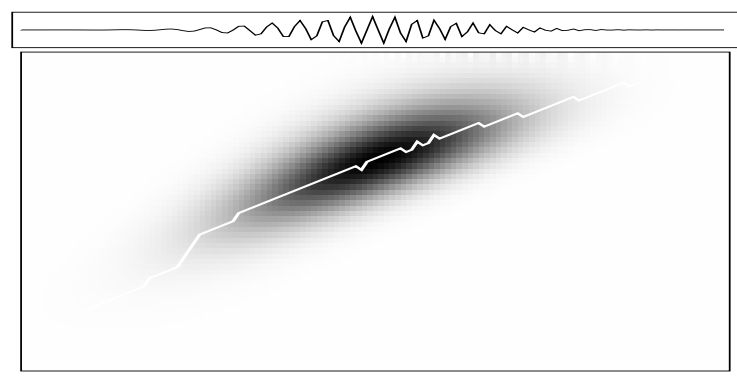

Figure 2: Chirp signal: ridge extraction by the annealing method.

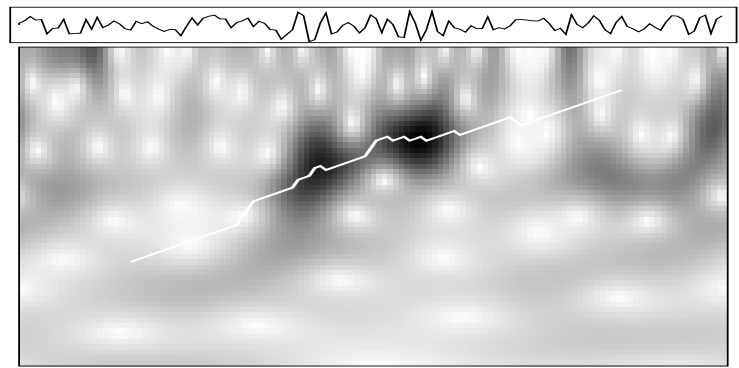

Figure 3: Noisy chirp signal: ridge extraction by the annealing method. 


\subsection{The snake approach}

The annealing approach described above has several limitations. In particular, the boundaries of the ridge are not handled in a systematic way: since one is looking for ridges of the form $a=\varphi(b)$, the boundary values of $b$ have to be fixed in advance (at least in the current form of the algorithm). One then has to know in advance the time duration of the signal under consideration (which is not a trivial problem in the noisy situation), or to introduce in the algorithm a post-processing stage to determine the actual location of the amplitude and frequency component under consideration.

An alternative algorithm may be based on the concept of "snakes" (or "active coutour models") developed in [11] in an image analysis context. In this second method the boundaries of the ridge adjust dynamically to the likely boundaries of the unknown ridge of the signal. In addition, this new algorithm can handle more complicated ridges. Finally, when combined with segmentation methods such as those proposed in [1] it could also handle multiple ridges. The new algorithm is then more adaptive. On the other hand, the price to pay is a loss of stability, and the latter can be significant in very noisy situations (see the discussion in [6].

2.3.1 Snake energy penalization Let us now consider a ridge as a parametrized curve in the time-frequency plane:

$$
r(s)=\left(\begin{array}{c}
\rho_{1}(s) \\
\rho_{2}(s)
\end{array}\right)
$$

where $s \in[0,1]$. The ridge then takes the form of a "snake" in the time-scale plane. To localize the snake near the location of the maxima of the modulus of the wavelet transform, we introduce a new cost function, based on the squared-modulus of the wavelet transform, as well as some additional terms needed in order to ensure the smoothness of the ridge (now understood as a smoothness requirement both in the $b$ and $a$ directions). Let us set:

$$
\begin{aligned}
F_{f}(r)= & -\int\left|T_{f}\left(\rho_{1}(s), \rho_{2}(s)\right)\right|^{2} d s \\
& +\int\left[\lambda_{a} \rho_{2}^{\prime}(s)^{2}+\mu_{a} \rho_{2}^{\prime \prime}(s)^{2}+\lambda_{b} \rho_{1}^{\prime}(s)^{2}+\mu_{b} \rho_{1}^{\prime \prime}(s)^{2}\right] d s
\end{aligned}
$$

where $\lambda_{a}, \lambda_{b}, \mu_{a}$ and $\mu_{b}$ are positive constants (in practice, these constante have to be adjusted to the overall normalization of the signal, and also to the number of nodes of the discretized snake). In the "snake terminology" of [11] the second term (containing the derivatives of $\rho_{1}$ and $\rho_{2}$ ) is the "internal energy" of the snake, and controls the smoothness and the rigidity of the snake. The first term is the "external energy" or the "image energy" of the snake, and accounts for the interaction of the snake with the wavelet transform modulus.

Remark: As before, when some spectral information on the noise is available, it may be incorporated into the cost function in the form of a renormalization potential $V(a)$, replacing $\left|T_{f}\left(\rho_{1}(s), \rho_{2}(s)\right)\right|^{2}$ by $\left|T_{f}\left(\rho_{1}(s), \rho_{2}(s)\right)\right|^{2}-V\left(\rho_{2}(s)\right)$.

Again the associated Euler equations are easily derived (see for instance [11] [5]). An iterative scheme was proposed in [11] for the numerical solution of these Euler, that has been widely used since then (see for example [1]). However, as before, such a scheme is 
efficient for smooth data, but not in the case of noisy signals. In addition, in the examples described in [11], they had to be started quite close to the actual solution, which is not adequate for the problem we address here. For that reason we turn again to simulated annealing procedures.

2.3.2 Cost minimization In practice, the snake is a discrete object. A discrete snake will be a collection of vectors of the form

$$
r=\left(\rho_{1}, \rho_{2}\right)=\left\{\left(\rho_{1}(0), \rho_{2}(0)\right),\left(\rho_{1}(1), \rho_{2}(1)\right), \cdots,\left(\rho_{1}(n-1), \rho_{2}(n-1)\right)\right\} .
$$

The integral in the cost function $F_{f}(r)$ is naturally replaced by a finite sum, taking into account the values of the snake at the nodes $0,1, \ldots n-1$ (see [11] for more details on the discrete formulation).

Starting from an initial guess $r_{0}=\left(\rho_{1,0}, \rho_{2,0}\right)$ for the ridge, we update it after each time step by randomly moving (by a given amount $\delta_{v}$ or $\delta_{h}$ ) a randomly chosen snake node. The neighborhood system is defined as follows: two neighboring ridges $r=\left(\rho_{2}, \rho_{2}\right)$ and $r_{1}^{\prime}=\left(\rho_{1}^{\prime}, \rho_{2}^{\prime}\right)$ can only differ at one point $\ell$ at most and in such a way that:

$$
\left\{\begin{array}{lll}
\rho_{2}^{\prime}(\ell)=\rho_{2}(\ell) \pm \delta_{v} & \text { and } \quad \rho_{1}^{\prime}(\ell)=\rho_{1}(\ell) & \text { or } \\
\rho_{2}^{\prime}(\ell)=\rho_{2}(\ell) & \text { and } \quad \rho_{1}^{\prime}(\ell)=\rho_{1}(\ell) \pm \delta_{h} &
\end{array}\right.
$$

More precisely, the general algorithm goes as follows ${ }^{3}$.

- Initialization: For a given initial value for the temperature, say $T_{0}=C / \ln (2)$, start from an initial guess for the ridge:

$$
r_{0}=\left\{\left(\rho_{1,0}(0), \rho_{2,0}(0)\right),\left(\rho_{1,0}(1), \rho_{2,0}(1)\right), \cdots,\left(\rho_{1,0}(n-1), \rho_{2,0}(n-1)\right)\right\}
$$

and compute the corresponding value $F_{f}\left(r_{0}\right)$ of the cost function.

- Step $k$ : Given the estimate at step $k-1$ of the ridge:

- Update the temperature (for instance with the schedule (14).

- Select randomly an integer $\ell \in[0, n-1]$ and a possible jump: left,right,up or down. Depending on the result, define the increments $\varepsilon_{v}=0$ or $\pm \delta_{v}, \varepsilon_{h}=$ 0 or $\pm \delta_{h}$. For instance, in the case of a left jump, the vertical increment $\varepsilon_{v}=0$ and the horizontal increment $\varepsilon_{h}=-1$, while $\varepsilon_{h}=1$ for a right jump. The candidate for the ridge at step $k$ is then:

$$
r_{k}^{c}=\left\{\left(\rho_{1, k}^{c}(0), \rho_{2, k}^{c}(0)\right),\left(\rho_{1, k}^{c}(1), \rho_{2, k}^{c}(1)\right), \cdots,\left(\rho_{1, k}^{c}(n-1), \rho_{2, k}^{c}(n-1)\right)\right\}
$$

where $\rho_{1, k}^{c}(j)=\rho_{1, k-1}(j)$ and $\rho_{2, k}^{c}(j)=\rho_{2, k-1}(j)$ for all $j \neq \ell$, and $\rho_{1, k}^{c}(\ell)=$ $\rho_{1, k-1}(\ell)+\varepsilon_{v}$ and $\rho_{2, k}^{c}(\ell)=\rho_{2, k-1}(\ell)+\varepsilon_{h}$.

- Compute the value of the cost function $F_{f}\left(r_{k}^{c}\right)$ and compare it with $F_{f}\left(r_{k-1}\right)$.

- If $F_{f}\left(r_{k}^{c}\right) \leq F_{f}\left(r_{k-1}\right)$ update the ridge: the new ridge at step $k$ is $: r_{k}=r_{k}^{c}$.

\footnotetext{
${ }^{3}$ We give the details of the algorithm in the case of the cost function $F_{f}(r)$ defined in (18). The same procedure can be used with the modified cost function $\tilde{F}_{f}(r)$.
} 
- Otherwise, pick a random number $\sigma$ uniformly distributed between 0 and 1 and:

$*$ if $\sigma \leq \exp \left\{-\left[F_{f}\left(r_{k}^{c}\right)-F_{f}\left(r_{k-1}\right)\right] / T_{k}\right\}$ update the ridge: $r_{k}=r_{k}^{c}$.

$*$ otherwise keep the previous ridge: $r_{k}=r_{k-1}$.

- Stopping criterion: When the ridge hasn't changed for a certain number of steps (fixed in advance), stop the iteration.

Again, a lot of variations around such a scheme are possible: the neighborhood system, the temperature schedule and the stopping criterion may be modified.

Remark: In the first method, we introduced a "smoothing + subsampling" procedure, in order to stabilize the algorithm by reducing its complexity and smoothing the data. In the case of the "snake" algorithm, the complexity reduction is automatically done by considering a limited number of nodes to describe the snake. However, it is still useful to smooth the wavelet transform modulus, to improve the stability of the algorithm.

\section{Signal reconstruction from the ridge}

We now want to achieve the characterization of the model signal, i.e. the reconstruction part. For the sake of simplicity we shall restrict ourselves to the case of a single ridge (the general case of finitely many arbitrary ridges is studied in [6]). We assume that the ridge can be parametrized as a continuous function: $\left[b_{\min }, b_{\max }\right] \ni b \hookrightarrow \varphi(b) \in(0, \infty)$. A good starting point could be the result of a ridge estimation procedure such as those presented in Section 2.1 and in Section 2.3. In any case, one will presumably only know few (sample) points:

$$
\left(b_{1}, \varphi_{1}\right),\left(b_{2}, \varphi_{2}\right), \cdots,\left(b_{n}, \varphi_{n}\right)
$$

and the smooth function $b \hookrightarrow \varphi(b)$ which we use is merely a guess which one constructs from the sample points. We use a smoothing spline (but any other kind of nonlinear regression curve would do as well).

\subsection{The problem}

The starting point of our analysis is again the heuristics inspired by the lemma 2, from which we expect that the restriction of the wavelet transform to its ridge (the so-called skeleton of the wavelet transform) reproduces the behavior of the signal itself (or more precisely that of its Hilbert transform, see [8] and [15]).

We assume that the values of the wavelet transform of an unknown signal of finite energy $f_{0}(t)$ are known at sample points $\left(b_{j}, \varphi_{j}\right)$ which are tentatively regarded as representative of the ridge of the modulus of the wavelet transform of the (unknown) signal $f_{0}(t)$.

$$
T_{f_{0}}\left(\left(b_{j}, \varphi_{j}\right)\right)=z_{j}
$$

The set of sample points $\left(b_{j}, \varphi_{j}\right)$ together with the values $z_{j}$ constitutes the skeleton of the wavelet transform of the signal to be reconstructed, and we use a smooth function $b \hookrightarrow \varphi(b)$ which interpolates the sample points as our best guess for the ridge of the modulus of the wavelet transform of $f_{0}$. 
We look for a signal $f(t)$ of finite energy whose wavelet transform $T_{f}(b, a)$ satisfies:

$$
T_{f}\left(b_{j}, \varphi_{j}\right)=z_{j}, \quad j=1, \cdots, n
$$

and has the graph of the function $\varphi(b)$ as ridge ${ }^{4}$.

The signal $f(x)$ solving this problem will be denoted $\hat{f}(x)$.

\subsection{Penalization}

We shall solve the reconstruction problem via the solution of an optimization problem.

We then look for a signal $f(t)$ whose wavelet transform $T_{f}(b, a)$ has the prescribed values at the sample points of the ridge, i.e. which satisfies the constraints $(21)$ while the $L^{2}$-norm in the scale variable $a$ (for $b$ fixed) of the modulus $M_{f}(b, a)=\left|T_{f}(b, a)\right|$ is kept to a minimum (this is meant to enforce the localization of the wavelet transform near the ridge). In other words we search for a signal $f(t)$ which minimizes the functional:

$$
F_{1}(f)=\frac{1}{c_{\psi}} \int d b \int \frac{d a}{|a|}\left|T_{f}(b, a)\right|^{2}=\|f\|^{2}
$$

while its wavelet transform has the prescribed values at the sample points of the ridge. Since the cost function $F_{1}(f)$ is a quadratic form in the unknown function $f$, the solution is easily computed by means of the use of Lagrange multipliers (see e.g. [3]). A solution is obtained as a linear combination of the wavelets $\psi_{\left(b_{j}, \varphi_{j}\right)}$ at the sample points of the ridge, the coefficients being given by the solution of a $n \times n$ linear system.

Such a reconstruction procedure is very similar to the reconstructions obtained by restricting the wavelet transform to its skeleton over its ridges, as suggested by lemma 2 .

However, this solution is not completely satisfactory, in particular, in the case where the sampling of the ridge is a coarse one. It also ignores the assumption that the restriction of the modulus $\left|T_{f}(b, a)\right|$ to the ridge is smooth and slowly varying. In order to force the solution of the constrained optimization problem to respect this requirement, we add the following contribution to the cost function:

$$
F_{2}(f)=\int_{b_{\min }}^{b_{\max }}\left(\left|\frac{d}{d b} T_{f}(b, \varphi(b))\right|^{2}-\frac{\omega_{0}^{2}}{\varphi(b)^{2}}\left|T_{f}(b, \varphi(b))\right|^{2}\right) d b
$$

viewed as an approximation of the more natural one, which is unfortunately not a quadratic form

$$
\tilde{F}_{2}(f)=\left.\int_{b_{\min }}^{b_{\max }}\left|\frac{d}{d b}\right| T_{f}(b, \varphi(b))\right|^{2} d b
$$

and consider the minimization of the cost function:

$$
\inf F(f)=\inf \left(F_{1}(f)+\epsilon F_{2}(f)\right)
$$

where the free parameter $\epsilon>0$ can be chosen to control the relative importance of the two contributions to the penalty. Consequently our reconstruction is given by the solution of the constrained optimization problem (25), subject to the linear constraints (21).

\footnotetext{
${ }^{4} \mathrm{An}$ alternative would consist in constraining only on the modulus of the wavelet transform at the sample points on the ridge, and to use the ridge information to reconstruct the phase; we won't discuss this approach here.
} 


\subsection{Solution of the minimization problem}

A simple computation shows that the cost function may be expressed as:

$$
F(f)=\iint Q(x, y) f(x) f(y) d x d y
$$

where the kernel $Q(x, y)$ is defined as follows:

$$
\begin{aligned}
Q(x, y)= & \delta(x-y)+\epsilon \int \frac{d b}{\varphi(b)^{4}}\left\{\psi\left(\frac{x-b}{\varphi(b)}\right)^{*} \psi\left(\frac{y-b}{\varphi(b)}\right)\left[\varphi^{\prime}(b)^{2}-\omega_{0}^{2}\right]\right. \\
& +\psi^{\prime}\left(\frac{x-b}{\varphi(b)}\right)^{*} \psi^{\prime}\left(\frac{y-b}{\varphi(b)}\right)\left[\frac{(x-b)(y-b)}{\varphi(b)^{2}}+1+\frac{x-2 b+y}{\varphi(b)}\right] \\
& +\psi\left(\frac{x-b}{\varphi(b)}\right)^{*} \psi^{\prime}\left(\frac{y-b}{\varphi(b)}\right) \varphi^{\prime}(b)\left[1+\frac{y-b}{\varphi(b)}\right] \\
& \left.+\psi^{\prime}\left(\frac{x-b}{\varphi(b)}\right)^{*} \psi\left(\frac{y-b}{\varphi(b)}\right) \varphi^{\prime}(b)\left[1+\frac{x-b}{\varphi(b)}\right]\right\} .
\end{aligned}
$$

(For the purpose of practical applications, the kernel $Q(x, y)$ becomes a finite matrix, whose entries can be computed using formula (26)).

From this point on the procedure introduced in [3] can be used to reconstruct the signal. Let us first introduce the pseudo-wavelets

$$
\widetilde{\psi_{j}}(x)=Q^{-1} \psi_{\left(b_{j}, \varphi_{j}\right)}, \quad j=1, \cdots, n .
$$

Then there exist complex numbers $\lambda_{1}, \cdots, \lambda_{n}$ (the Lagrange multipliers of the problem) such that the solution $\hat{f}$ of the optimization problem is given by the formula:

$$
\hat{f}(x)=\sum_{j=1}^{n} \lambda_{j} \widetilde{\psi_{j}}(x)
$$

Defining the matrix $\mathcal{M}$ by

$$
\mathcal{M}_{k \ell}=\left\langle\widetilde{\psi_{k}}, \psi_{\left(b_{\ell}, \varphi_{\ell}\right)}\right\rangle=\left\langle Q^{-1} \psi_{\left(b_{j}, \varphi_{j}\right)}, \psi_{\left(b_{\ell}, \varphi_{\ell}\right)}\right\rangle
$$

we finally obtain the values of the Lagrange multipliers as follows

$$
\lambda_{j}=\sum_{\ell}\left(\mathcal{M}^{-1}\right)_{j \ell} z_{\ell}
$$

The results of the discussion of this section can be summarized in the following algorithm.

1. determination of a sampling $\left(b_{1}, \varphi_{1}\right), \cdots,\left(b_{n}, \varphi_{n}\right)$ of the ridge,

2. construction of a smooth estimate $b \hookrightarrow \varphi(b)$ of the ridge from the sample points, for instance via spline interpolation,

3. computation of the matrix $Q(x, y)$ of the penalty along the ridge estimate, 
4. computation of the reconstruction wavelets $\hat{\psi}_{j}=Q^{-1} \psi_{b_{j}, \varphi_{j}}$ at the ridge sample points of the time-scale plane,

5. computation of the coefficients $\lambda_{j}$.

The solution $\hat{f}$ to the reconstruction problem is then given by formula (28).

As we shall see in the next illustrations, the matrix of the kernel $Q(x, y)$ has a very simple structure. It is then very likely that it may be computed in an efficient way. This would speed up steps 3 and 4 of the reconstruction algorithm (these steps are the most time consuming part of the algorithm; in particular we used a numerical integration procedure for the illustration of the next section).

Remark: An alternative to the penalty (22) is given by the following one

$$
\widetilde{F}_{1}(f)=\frac{1}{c_{\psi}} \sum_{k=1}^{n} \int \frac{d a}{|a|}\left|T_{f}\left(b_{k}, a\right)\right|^{2}
$$

that only uses the samples of the ridge. The advantage of such a formula is that it does not penalize the modulus of the wavelet transform in between ridge samples.

\subsection{Illustration}

To illustrate the method, we present in figures 4 and 5 the chirp signal reconstructed from the ridge using the above described procedure (figure 4), and the difference between the reconstructed signal and the original (figure 5). The signal (see figure 1) is a 128 samples one, and the number of values of the wavelet transform used here (i.e. the number of samples $\left.\left(b_{k}, \varphi\left(b_{k}\right)\right)\right)$ is of the order of 30 . The quality of reconstruction is quite good (the order of magnitude of the error is seen to be about $2 \%$ ).

The kernel $Q(x, y)$ (see (26)) was evaluated using a standard Romberg numerical integration routine and its modulus is displayed (coded with gray levels) in figure 6 . It is interesting to notice that the kernel has a very simple shape $(Q(x, y)-\delta(x-y)$ is diagonal dominant, as well as $Q(x, y)$ and $\left.Q^{-1}(x, y)\right)$. It is then very likely that its numerical evaluation can be performed efficiently using adapted methods, see [2]). In addition, the complete kernel is in general a perturbation of the identity, so that for $\epsilon$ small enough, standard iterative methods may be used to evaluate $Q^{-1}$.

Again, more detailed illustrations may be found in [5].

\section{Final remarks}

Let us stress that the "ridge identification + reconstruction" algorithm may be called an adaptive one. Indeed, it only uses information from the region in the time-frequency plane where the signal is localized. No threshold is needed here; the algorithm in some sense forces to zero the irrelevant regions of the time-frequency plane, as would do the non-linear thresholding methods.

Let us also stress that the method is really dedicated to chirp-like signals, i.e. signals that may be characterized by a ridge. Similar methods may probably be adapted to other situations, such as singular or transient signals (see [4]). 


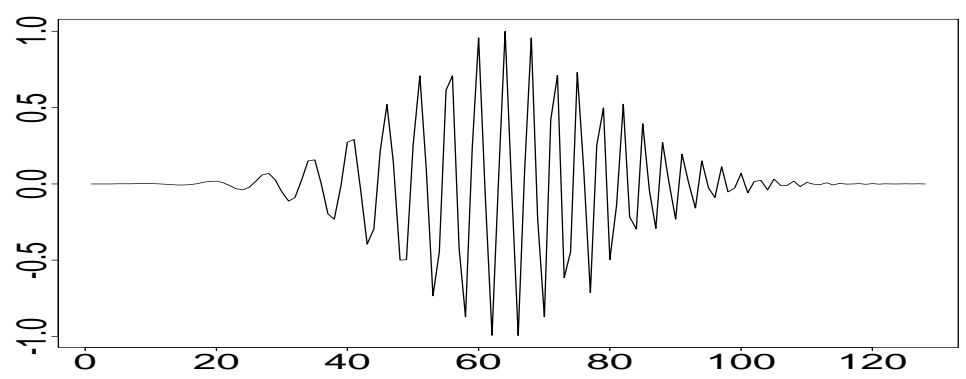

Figure 4: Chirp signal reconstructed with the above procedure.

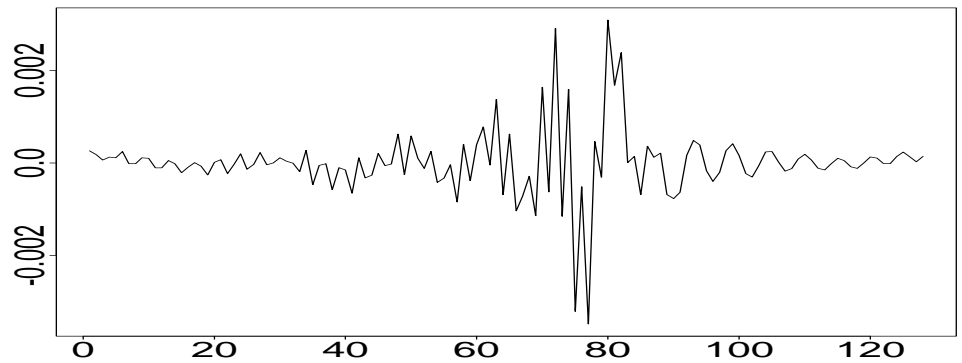

Figure 5: Difference between original and reconstructed chirp signals.

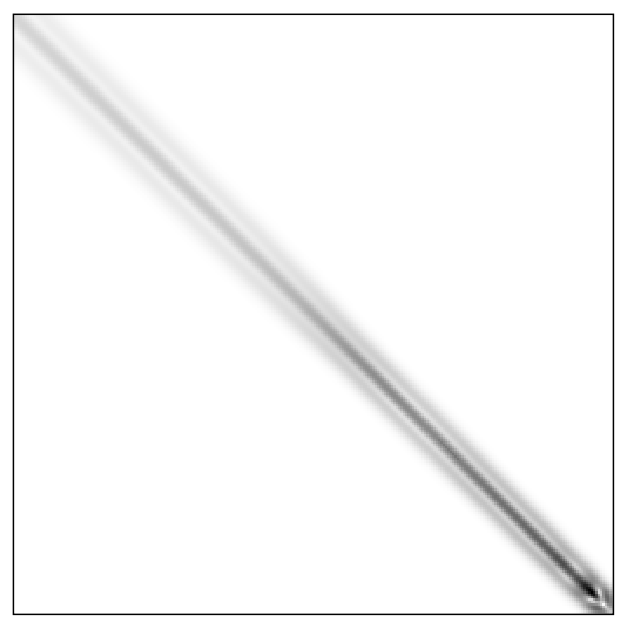

Figure 6: Matrix representation of the kernel $|Q(x, y)-\delta(x-y)|$. 


\section{References}

[1]Berger, M.-O., Towards Dynamic Adaptation of Snake Contours, in International Conference on Image Analysis and Processing, Como (Italy), IAPR, september 1991 (1993).

[2]Beylkin, G., Coifman, R. and Rokhlin, V., Fast wavelet transforms and numerical algorithms I. Comm. Pure and Appl. Math., 44 (1991) 141-183.

[3]Carmona, R., Extrema Reconstruction and Spline Smooting: Variations on an Algorithm of Mallat and Zhong (this volume).

[4]Carmona, R., Wavelet Identification of Transients in Noisy Signals, Proc. SPIE 15-16 June 1993, San Diego, California. Mathematical Imaging: Wavelet Applications in Signal and Image Processing. 392-400.

[5]Carmona, R., Hwang, W.L., and Torrésani, B., Characterization of Signals by the Ridges of their Wavelet Transform, preprint (1994).

[6]Carmona, R., Hwang, W.L., and Torrésani, B., Ridge Detection and Speech Reconstruction, preprint (1994).

[7]Daubechies, I., Ten Lectures on Wavelets. SIAM (1992).

[8]Delprat, N., Escudié, B., Guillemain,P., Kronland-Martinet, R., Tchamitchian, Ph., Torrésani, B., Asymptotic wavelet and Gabor analysis: extraction of instantaneous frequencies. IEEE Trans. Inf. Th. 38, special issue on Wavelet and Multiresolution Analysis (1992) 644-664.

[9]Flandrin, P., Temps-Fréquence. Traité des Nouvelles Technologies, série Traitement du Signal, Hermes (1993).

[10]Geman, S., and Geman, D., Stochastic Relaxation, Gibbs Distributions and Bayesian Restoration of Images. IEEE Proc. Pattern Ana. Mach. Intell. 6 (1984), 721-741.

[11]Kass, M., Witkin, A., and Terzopoulos, D., Snakes: Active Contour Models, Int. J. of Computer Vision,(1988) 321-331.

[12]van Laarhoven, P.J.M., and Aarts, E.H.L., Simulated Annealing: Theory and Applications, Reidel Pub. Co. (1987).

[13]McAulay, R.J., and Quatieri, T.F., Low Rate Speech Coding Based on the Sinusoidal Model. In Advances in Speech Signal Processing, Edited by S. Furui and M. Mohan Sondui (1992).

[14]Mallat, S., and Zhong, S., Characterization of Signals from Multiscale Edges. IEEE Trans. Pattern Anal. Machine Intel., (1991).

[15]Tchamitchian, Ph., and Torrésani, B., Ridge and Skeleton Extraction from the Wavelet Transform. In Wavelets and Applications, M.B. Ruskai \& al Eds, Jones \& Bartlett Pub. Comp., Boston, (1992) 123-151.

[16]Ville, J., Théorie et Applications de la Notion de Signal Analytique, Cables et Transmissions 2ème A (1) (1948) pp. 61-74. 